\title{
Von Sparmassnahmen und Haftungsrisiken
}

\section{Eva Druey Just}

Dr. iur., Rechtsanwältin in Chur

\author{
Die Diskussion um Kostendämpfungsmassnahmen im Gesundheitswesen wurde \\ im vergangenen Herbst neu angeheizt durch einen Katalog von Vorschlägen einer \\ Expertengruppe des EDI. Viele dieser Massnahmen bergen zusätzliche Haftungs- \\ risiken - ein Umstand, der in der Diskussion um Sparziele bis anhin viel zu wenig \\ Beachtung fand.
}

\section{Die Bemühungen um Kosteneindämmung}

\section{Rationierung und Rationalisierung}

Seit Jahren bemühen sich alle Akteure des Gesundheitswesens um Kostensenkungen. Es gilt, Unnötiges ohne Qualitätsverlust zu beseitigen und Abläufe effizienter zu gestalten [1]. Jüngst wurde die Diskussion durch eine interdisziplinäre Expertengruppe des EDI um einen Strauss neuer Ideen zur Kostensenkung bereichert, reichend bis hin zu einschneidenden Änderungen wie der Einführung von Globalbudgets [2]. Die durchaus erstrebenswerte Rationalisierung steht dabei im Gegensatz zur Rationierung: Hier wird nicht bloss die Effizienz gesteigert, sondern es werden Leistungen eingeschränkt, welche aus medizinischer Sicht notwendig wären. Unumgängliche Folge ist eine Quali-

Die Sammlung von Ideen zur Kosteneinsparung im Gesundheitswesen ist in vollem Gange. Zahlreiche Massnahmen, wie beispielsweise eine vermehrte Planung des Angebotes, die Senkung von Arzneimittelpreisen oder die Erhöhung des Preisdrucks, sind bereits in Umsetzung begriffen. Ideen wie die Einführung von Globalbudgets, die konsequente Bevorzugung ambulanter vor stationären Eingriffen oder die Verstärkung der Rechnungskontrolle werden diskutiert. Kaum zur Sprache kommt in dieser Diskussion, dass die vorgeschlagenen Massnahmen die Haftungsrisiken der Leistungserbringer gleich in mehrfacher Hinsicht verschärfen. Diese erhöhten Risiken werden nicht folgenlos bleiben. Sie führen entweder dazu, dass die Sparmassnahmen umgangen werden, oder aber zu einer deutlichen Erhöhung der Prämien von Haftpflichtversicherungen. Beides bedeutet letztlich einen Rückschritt auf dem Weg zur Kostendämpfung. Die Akteure im Gesundheitswesen tun gut daran, bei der Diskussion um Einsparungen die Haftpflichtproblematik nicht ganz beiseite zu lassen. tätseinbusse. Die Rationierung widerspricht einerseits den Zielen des KVG [3]. So verlangt der Art. 43 Abs. 6 KVG die "qualitativ hoch stehende und zweckmässige gesundheitliche Versorgung zu möglichst günstigen Kosten». Anderseits schuldet der Arzt seinem Patienten Qualität in Form einer sorgfältigen, den Regeln der ärztlichen Kunst folgenden Behandlung. Es entsteht ein Spannungsverhältnis von Kostensenkung und Qualitätspflichten. Wie sich dieses auf die Haftpflichtrisiken auswirken kann, wird nachfolgend kurz dargestellt.

\section{Bekanntes und Neues an Kosten- dämpfungsmassnahmen}

\section{Bekannte Massnahmen}

Die auf politischem Weg bereits realisierten Massnahmen [4] fokussieren auf eine verstärkte Planung des Angebotes, insbesondere durch Spitalplanung und Zulassungsbeschränkungen für Arztpraxen. Daneben erfolgten Verbilligungen von Arzneimitteln sowie Eingriffe in die Tarifstruktur TARMED, welche fehlerhafte Anreize und Übervergütungen vermeiden sollen [5].

Weniger auf politischem als vielmehr auf gerichtlichem Weg wurden Preissenkungen erreicht. Im ambulanten Bereich mussten Spitäler zuletzt empfindliche Mindervergütungen erdulden. Auch die Basisfallpreise für stationäre Behandlungen liegen heute tendenziell tiefer als noch bei Einführung der Fallpauschalen im Jahr 2012 [6]. Als spektakuläre gerichtliche Bemühung zur Kostensenkung darf auch das Myozyme-Urteil des Bundesgerichts genannt werden [7]. Das Bundesgericht 
liebäugelt in den Erwägungen zu diesem Urteil mit der sogenannten "QALY»-Methode, ohne diese aber konkret gutzuheissen oder abzulehnen. Nach der QALYMethode könnten die Behandlungskosten für einen Patienten auf einen bestimmten Betrag - im Gespräch war eine Summe von CHF 100000 pro gewonnenem Lebensjahr von hoher Lebensqualität («quality-adjusted life year» oder "QALY») - limitiert werden.

Die geschilderten Massnahmen bewegen sich mehrheitlich im Bereich der Rationalisierung. Rationierungen, also konkrete Leistungs- oder Qualitätseinschränkungen, sind nur vereinzelt feststellbar. So ist die im TARMED 1.09 per 1. Januar 2018 erfolgte Limitierung einer normalen Konsultation auf 20 Minuten streng genommen eine Rationierung, die aber bei erhöhtem Behandlungsbedarf Ausnahmen kennt und daher medizinisch keine negativen Auswirkung haben sollte. Versteckte Rationierungen drohen, wenn die Preise so weit gesenkt werden, dass eine kostendeckende Leistungserbringung auch bei hoher Effizienz nicht mehr möglich ist. Ganz eindeutige Rationierungen würde eine Umsetzung der QALY-Methode mit sich bringen, die allerdings bis heute weder politisch noch auf gerichtlichem Weg konsequent erfolgt ist.

\section{Neue diskutierte oder geplante Massnahmen}

Das gesamte Spektrum geplanter oder angedachter Massnahmen zu diskutieren, ginge an dieser Stelle zu weit. Erwähnt werden nur einige, die aktuell für besonders hitzige Diskussionen sorgen [8]:

- Globalzielvorgaben für das Kostenwachstum der obligatorischen Krankenpflege (OKP): Das akzeptierte Wachstum der Gesundheitsausgaben wird fixiert. Nach dem im Raum stehenden Vorschlag soll für die vier OKP-Kostenblöcke Spitäler, freipraktizierende Ärzte, Arzneimittel und Übrige je ein Budget aufgestellt werden, bei dessen Überschreitung Sanktionen drohen. Ist das Jahresbudget erreicht, muss eine Behandlung auch dann in die nächste Budgetperiode verschoben werden, wenn sie medizinisch indiziert ist. "Nötige Leistungen» [9] sollen weiterhin erbracht werden können.

- Ambulant vor stationär: Einfache Eingriffe, für die eine stationäre Aufnahme nicht notwendig ist, sollen zwingend ambulant durchgeführt werden. Die Kantone Zürich und Luzern haben bereits Listen solcher Eingriffe erlassen, der Bund wird per 1. Januar 2019 nachziehen [10].

- Health Technology Assessment (HTA): Der Pflichtleistungskatalog soll überprüft und Leistungen allenfalls von der OKP ausgeschlossen bzw. nur noch bei strenger Prüfung der Indikation übernommen werden.
- Rechnungskontrolle: Bereits heute vergütet die OKP nur Leistungen, welche den Kriterien der Wirksamkeit, Zweckmässigkeit und Wirtschaftlichkeit («WZW-Kriterien») standhalten. Werden bereits erbrachte Behandlungen im Nachhinein als WZWwidrig erachtet, verweigert die Versicherung die Vergütung. Diese Kontrolle soll verstärkt werden. Die vermehrte Einholung von Kostengutsprachen wird notwendig sein.

Die diskutierten Massnahmen gehen stärker als die bereits umgesetzten in Richtung Rationierung. Insbesondere die Globalziele, aber auch die weiteren Massnahmen nehmen in Kauf, dass Leistungen trotz medizinischer Indikation nicht erbracht werden können. Vor allem die drei letztgenannten Massnahmen greifen in die ärztliche Behandlungshoheit ein und übertragen konkrete Behandlungsentscheidungen auf Krankenversicherer, Vertrauensärzte oder den Pflichtleistungskatalog.

\section{Kostendämpfung und Haftungsrisiken}

\section{Ärztlich geschuldete Sorgfalt}

Ärzte schulden ihren Patienten die gewissenhafte Ausübung der ärztlichen Kunst. Sie haben ihre Patienten «fachgerecht zu behandeln, zum Schutze ihres Lebens oder ihrer Gesundheit insbesondere die nach den Umständen gebotene und zumutbare Sorgfalt zu beachten, grundsätzlich folglich für jede Pflichtverletzung einzustehen" [11]. Im Einzelfall massgeblich ist jeweils die berechtigte Erwartung der Patienten an die Behandlung [12]. Wird die geschuldete Sorgfalt nicht erbracht, drohen Schadenersatzforderungen und strafrechtliche Folgen. Die Rechtsprechung zur geschuldeten Sorgfalt gilt seit Jahren unverändert. Kostenüberlegungen sind ihr weitgehend fremd.

\section{Die Vereinbarkeit von Sparmassnahmen mit dem geltenden Haftpflichtrecht}

Kostensenkungsmassnahmen als entlastendes Argument im Schadensfall

Die Bemühungen um Kosteneinsparungen der vergangenen Jahre haben die Rechtsprechung zum Haftpflichtrecht schon deswegen kaum beeinflussen können, weil die Haftpflicht eine Angelegenheit zwischen Arzt und Patient ist. Demgegenüber betreffen die Sparmassnahmen stets die Finanzierungsseite. Diese beschlägt nach unserem System in der Regel nicht das Verhältnis Leistungserbringer-Patient, sondern dasjenige des Leistungserbringers zur obligatorischen Krankenversicherung. 
Wenn Kostendämpfungsmassnahmen Rationierungscharakter erhalten, können die Regeln ärztlicher Kunst möglicherweise nicht mehr eingehalten werden. Kommt es deswegen zu einem Haftpflichtfall, müssen Ärzte und Spitäler versuchen, sich unter Anführung der angeordneten Massnahme von der Haftpflicht zu entlasten. Dies bedeutet, dass sie Vorgaben aus der Rechtsbeziehung zur Krankenversicherung in ihre Rechtsbeziehung zum Patienten tragen müssen. Es wurde oben gesagt, dass für die geschuldete Sorgfalt die berechtigte Erwartung des Patienten massgeblich ist. Die Entlastung wird daher gelingen, wenn der Patient die Vorgabe kennt und seine Erwartungen entsprechend anpassen muss. So wird sich ein Patient kaum darüber beklagen können, dass eine Operation ambulant durchgeführt wurde, wenn eine kantonale Liste die stationäre Aufnahme verbietet. Dasselbe gilt für die Unterlassung von Behandlungen und Medikationen, welche von den Pflichtleistungen ausgeschlossen wurden. Die Pflicht von Arzt und Spital wird es in solchen Fällen sein, die Patienten über die entsprechende Vorgabe zu orientieren, um die berechtigten Erwartungen zu dämpfen.

\section{Risiken bei der Prioritätensetzung}

Die Entlastung vom Vorwurf der unsorgfältigen Behandlung wird demgegenüber dann sehr schwierig, wenn eine Sparmassnahme nicht genügend greifbar ist, um als Anweisung für eine konkrete Behandlung verstanden zu werden. Dies ist der Regelfall. Das Beispiel mit der Liste ambulanter Eingriffe wird die Ausnahme bleiben. Die meisten Sparmassnahmen führen letztlich schlicht zu gekürzten Budgets. Was in dem Budget Platz haben muss, bleibt die Entscheidung der Leistungserbringer. Mit anderen Worten: Sie müssen Prioritäten setzen. Steigen bei gekürztem Kostenrahmen die Wartezeiten auf der Notfallstation, muss die Triage umso besser sein. Führt ein Globalbudget zu Wartefristen für elektive Eingriffe, müssen die Folgen des Abwartens abgeschätzt werden. Darf eine Konsultation nur zwanzig Minuten dauern, muss der absolut notwendige Inhalt definiert werden. Gekürzte Budgets geben mit anderen Worten der ärztlichen Pflicht zur Setzung von Prioritäten eine ganz neue Bedeutung. Die Sortierung von Nötigem, Wünschenswertem und Unnötigem ist fraglos effizienzsteigernd, aber schadengeneigt. Gerade im Fall von Globalzielen oder anderen Budgetvorgaben wird sie allein in die Verantwortung der Leistungserbringer geschoben.

Risiken durch Verlagerung der Behandlungsverantwortung Ein weiteres Risiko bergen Sparbemühungen dort, wo Teile der Behandlungsverantwortung an die Kranken- versicherungen übergehen, namentlich bei der vorgeschlagenen Stärkung der Rechnungskontrolle. Aus der Perspektive des Haftpflichtrechts ist diese Verschiebung von Entscheidungskompetenzen problematisch, da der Arzt zwar Vorgaben der Versicherungen erfüllen muss, gegenüber dem Patienten aber verantwortlich bleibt. Die Zürcher Sozialversicherungsrichterin Brigitte Pfiffner Rauber geht zwar in ihrer Dissertation zu Recht davon aus, dass das Parlament wenigstens für die Vertrauensärzte dieselbe Behandlungsverantwortung vorgesehen hatte wie für die Behandelnden: Zwei Minderheitsanträge wurden «abgelehnt mit der Begründung, dass die bestehenden zivil- und strafrechtlichen Haftungsnormen auch gegenüber Vertrauensärztinnen und -ärzten Geltung hätten" [13]. Doch ist diese Bestimmung soweit ersichtlich toter Buchstabe geblieben. Letztlich ist und bleibt es der Arzt und nicht die Kasse, welcher seinem Patienten die medizinisch korrekte Behandlung schuldet. Eine Krankenversicherung kann schon nach geltendem Recht eine Kostengutsprache verweigern, ohne ernstlich mit Haftpflichtansprüchen rechnen zu müssen. Hält der Arzt die verweigerte Behandlung dennoch für notwendig, muss er dem Patienten empfehlen, sie auf eigene Kosten vornehmen zu lassen. Besonders dann, wenn Gefahr im Verzug und eine Finanzierung dem Patienten nicht zumutbar ist, bleibt dem Behandelnden die Wahl zwischen Pest und Cholera: Wird die Behandlung vorgenommen, droht die Rechnung dafür offen zu bleiben. Wird sie nicht vorgenommen, drohen Haftpflichtansprüche der Patienten wegen Unterlassung einer notwendigen Behandlung.

\section{Fazit: Vergesst nicht die Haftpflicht!}

Die Analyse der umgesetzten, geplanten und diskutierten Kostendämpfungsmassnahmen zeigt, dass praktisch jede von ihnen das Potential hat, die Haftungsrisiken der Leistungserbringer zu erhöhen. Je vager die Vorgabe, desto grösser ist die Verantwortung der Ärzte bei der Priorisierung der noch erbringbaren Leistung, desto grösser die Fehleranfälligkeit bei der Setzung der Prioritäten. Wird die Vorgabe konkreter, gibt sie den Leistungserbringern eher Argumente, um sich von ihrer Haftpflicht zu befreien. Es trifft sie aber die Pflicht, ihre Patienten über die Rationierung aufzuklären. Zudem haben konkrete Behandlungsvorgaben den Nachteil, dass sie mit einer Einschränkung der ärztlichen Behandlungshoheit einhergehen.

Die Diskussion um Sparbemühungen muss daher immer mit Blick auf das Haftpflichtrecht geführt werden. Geschieht dies nicht, steigen die Haftungsrisiken. Die Leistungserbringer werden dem entweder begegnen, 
indem sie Sparmassnahmen bekämpfen und vielleicht umgehen. Oder sie werden die gesteigerten Risiken auf sich nehmen, was zu deutlich steigenden Haftpflichtversicherungsprämien führen dürfte. Diese wiederum stellen einen neuen Kostenfaktor dar. Eine Vernachlässigung der Haftungsrisiken bei der Festlegung von Sparmassnahmen bedeutet somit stets eine ganze oder teilweise Vereitelung der Sparziele.

\section{Interessenverbindungen}

Im Rahmen ihrer anwaltlichen Tätigkeit berät und vertritt Eva Druey Just verschiedene Leistungserbringer des Gesundheitswesens.

\section{Literatur}

1 Unabhängige interdisziplinären Arbeitsgruppe «Gerechte Ressourcenverteilung im Gesundheitswesen": Manifest für eine faire Mittelverteilung im Gesundheitswesen, Zürich, 2. Auflage 2006, 6; Pfiffner Rauber B: Das Recht auf Krankheitsbehandlung und Pflege; Zum Behandlungsanspruch von Krankenversicherten im Rahmen der Wirtschaftlichkeit unter besonderer Berücksichtigung der Langzeitpflege, Forum Gesundheitsrecht Band 8, Zürich 2003, S. 221f.

Dr. Eva Druey Just Rechtsanwältin VINCENZ \& PARTNER Tel. +41812585594 e.druey[at] vincenzpartner.ch www.vincenzpartner.ch

2 Zusammengefasst im Bericht vom 24.8.2017 der Expertengruppe des EDI, Kostendämpfungsmassnahmen zur Entlastung der obligatorischen Krankenpflegeversicherung, abrufbar unter https:// www.bag.admin.ch/bag/de/home/themen/versicherungen/krankenversicherung/kostendaempfung-kv.html.
3 So schrieb der Bundesrat in der Botschaft zum KVG 1992: «Der hier gewählte Weg gestattet, eine qualitative und quantitative Leistungsrationierung zu vermeiden»: BBl 1992 I 93, 96 und 133.

4 Siehe die Expertengruppe des EDI, zitiert in FN 2.

5 Siehe Bundesamt für Gesundheit, Faktenblatt «Anpassungen des Ärztetarifs Tarmed vom 16. August 2017» sowie die dazu erlassene Medienmitteilung des BAG vom 16.8.2017.

6 Mindervergütungen im TARMED erfolgten auch über den Eingriff in die Tarifstruktur, siehe FN 5 oben. Beispiele für die Senkung ambulanter Preise durch das Bundesverwaltungsgericht: Urteile des Bundesverwaltungsgerichts C-4505/2013, C-2380/2012 C-1220/2012, C-3175/2013; In der Tendenz sinkende Basisfallpreise im stationären Bereich lassen sich nachverfolgen via Schweizerische Konferenz der kantonalen Gesundheitsdirektorinnen und -direktoren, Übersicht über die kantonalen Spitallisten und Spitaltarife, Stand 10.8.2017, auch online abrufbar über www.gdk-cds.ch.

7 BGE 136 V 392; dazu z.B. Wasserfallen J-B/Junod V, Rationierung der Behandlungen: Was bedeutet der Bundesgerichtsentscheid «Myozyme»?, SÄZ. 2011;92:1751ff.

8 Bericht vom 24.8.2017 der Expertengruppe des EDI, FN 2.

9 Zit. Bericht vom 24.8.2017 der Expertengruppe des EDI, FN 2, S. 30.

10 Gemeinsame Medienmitteilung der Gesundheitsdirektion des Kantons Zürich und des Gesundheits- und Sozialdepartements des Kantons Luzern vom 26.10.2017, abrufbar unter https://gd.zh.ch/ internet/gesundheitsdirektion/de/aktuell/medienmitteilungen. html; Änderung der Krankenpflege-Leistungsverordnung: AS 2018 967f.; weitere Kantone planen Ähnliches.
11 BGE 120 Ib 411 E. 4 a.

12 Urteil des Bundesgerichts 4C.53/2000 E. 1 b.

13 Pfiffner Rauber B, Endnote 1 oben, S. 148 Fn 50, unter Verweis auf Amtl. Bulletin SR vom 17.12.1992, S. 1324 (Verhandlungsheft S. 218). 